\title{
Estudio acerca de las pruebas de evaluación del E/LE en el contexto educativo gabonés ${ }^{1}$
}

\section{Study on E/LE assessment tests in the Gabonese educational context}

DOI: $10.46932 /$ sfjdv2n4-082

Received in: March 1st, 2021

Accepted in: May 30th, 2021

\author{
Stéphanie Messakimove \\ Enseignant-chercheur \\ École Normale Supérieure / CRAAL Libreville, Gabon \\ E-mail: masthebony@yahoo.fr
}

\section{RESUMEN}

Los motivos que nos han llevado a conocer las convergencias y divergencias de los criterios de evaluación manifiestos en unas pruebas y otras son tres: (1) la ausencia de estudios sobre el desempeño de los alumnos en la prueba escrita de español en el Bachillerato; (2) la percepción de que existen diferencias sustanciales en el entrenamiento en función del perfil y las prácticas de cada instituto educativo; y (3) las carencias formativas de los profesores en cuestiones de evaluación. Con esta investigación, pretendíamos contribuir a enriquecer la reflexión y forma de mejorar tanto el desempeño de los alumnos en las pruebas de Bachillerato como la práctica de E/LE en Gabón. Para concretar mejor esta pretensión genérica, formulamos dos objetivos generales que permitieron la delimitación de la investigación. En el primer objetivo, se pretendía analizar la prueba escrita de español que es utilizada para medir los resultados alcanzados por los alumnos al final de los estudios secundarios; Pero también es utilizada por los docentes para conocer qué aspectos de la lengua domina el alumno y cuáles no. Con el segundo objetivo, necesitamos determinar si las pruebas diseñadas y aplicadas en los centros escolares para medir el desempeño de los alumnos se ajustan a la normativa que la regula.

Palabras clave: Evaluación, examen escrito, universidad, educación comparada.

\begin{abstract}
The reasons that have led us to learn about the convergences and divergences in the evaluation criteria shown in some tests and others are threefold: (1) the absence of studies on the performance of students in the written Spanish test in high school; (2) the perception that there are substantial differences in training depending on the profile and practices of each educational institute; and (3) the lack of training of teachers in evaluation issues. With this research, we intended to contribute to enrich the reflection and ways to improve both the performance of students in the baccalaureate tests and the practice of E/LE in Gabon. In order to better specify this generic pretension, we formulated two general objectives that allowed the delimitation of the research. In the first objective, we intended to analyze the written Spanish test that is used to measure the results achieved by students at the end of secondary school; but it is also used by teachers to know which aspects of the language the student masters and which ones he/she does not. With the second objective, we need to determine whether the tests designed and applied in schools to measure student performance are in accordance with the regulations that govern them.
\end{abstract}

Key words: Assessment, written test, university, comparative education.

\footnotetext{
${ }^{1}$ En este artículo, la autora presenta los avances de la Tesis de Doctorado, “Análisis del desempeño de los alumnos gaboneses en la prueba escrita de español en el bachillerato: Implicaciones para la enseñanza de ELE en Gabón”, que está realizando.
} 


\section{INTRODUCCIÓN}

La idea de esta investigación sobre el desempeño de los alumnos en la prueba escrita de español (PEE) tiene su origen en nuestra experiencia como profesora y examinadora en el examen de Bachillerato $^{2}$. Durante este periodo, hemos podido observar que los resultados alcanzados por los alumnos difieren ampliamente en función de la titularidad de los centros ( $c f$. Rapports de la Direction Générale $d u$ Baccalauréat, 2009-2013). La Nota de información Nº02 del 24/09/2011 establece que la PEE tiene que adecuarse a las orientaciones oficiales por tener carácter común y obligatorio en todo el país. Como evaluación, final la PEE valora el rendimiento de los alumnos al final del proceso de aprendizaje: "lo que han aprendido", "lo que saben" o "lo que pueden hacer" (Bordón 2006, p. 19).

Sin embargo, ¿en todos los institutos se aplica la normativa que regula la PEE? ¿Están los alumnos entrenados en lo mismo?

En el ámbito de la adquisición de segundas lenguas (ASL), el tema de la evaluación ha sido abordado desde varias perspectivas generando una abundante literatura (Bordón, 2015; Sanmartí Puig, 2020; Pastor Cesteros, 2003, entre otros). En didáctica de L2/LE, la noción de evaluación varía según el método o enfoque elegido, puesto que las prácticas de evaluación tienen que ser inseparables de las prácticas pedagógicas. Cuando se toma una opción de metodología didáctica, se está tomando, aunque sea implícitamente, una decisión de evaluación.

En el campo educativo, la evaluación permite descubrir que los objetivos planteados se han cumplido o no, lo que servirá para retomar aquello que no fue asimilado por los alumnos, reforzar los éxitos obtenidos y no incurrir en los mismos errores en el futuro, así poder introducir el cambio de estrategias pedagógicas para enmendar lo insuficiente. Desde una visión abarcadora del concepto de evaluación podemos entender que se trata de una operación indisociable de todo proceso de enseñanza/aprendizaje, al margen de su materialización o no en según qué tipo de pruebas (Pastor Cesteros, 2003; Marín Perís, 2008).

El tema de los resultados de los alumnos en la PEE en el Bachillerato es un punto problemático para las autoridades educativas y formadores de formadores. Las últimas reformas curriculares para la mejora de la calidad de la educación en su conjunto $(2002,2005,2011,2013,2014)$ y las del Baccalauréat $^{3}$ (2011, 2013, 2014) no han dado los resultados esperados. Se sigue registrando resultados poco satisfechos en las pruebas escritas oficiales. Una situación que genera varias interrogantes tanto

\footnotetext{
${ }^{2}$ En este trabajo, utilizaremos la palabra Bachillerato (con mayúscula) para referirse al diploma del Baccalauréat gabonés y, bachillerato (con minúscula) para señalar el nivel escolar que finaliza los estudios de la enseñanza secundaria ( $2^{\circ}$ curso de bachillerato) y que corresponde al nivel Terminale del sistema educativo gabonés

${ }^{3}$ En el sistema educativo gabonés el Baccalauréat es el conjunto de exámenes que sirven para obtener el título de bachillerato al final de la enseñanza secundaria y que son condición indispensable para acceder a la universidad. Son dos grupos de pruebas (10 u 11 asignaturas) organizadas sucesivamente.
} 
sobre el proceso de entrenamiento de los alumnos como lo que se mide en las diferentes pruebas de evaluación y su fiabilidad.

Algunas investigaciones (Caro Muñoz, 2017; Eyeang, 1997, 2011; Mbadinga Mbadinga, 2014; Messakimove, 2021) han señalado la complejidad del contexto gabonés de E/A de E/LE que influye tanto en las prácticas docentes como en los resultados alcanzados por los alumnos. De manera general todos apuntan una multitud de factores contextuales que inciden en el complejo fenómeno de aprender E/LE: presencia de la didáctica francesa, falta de infraestructuras, materiales didácticos insuficientes y/o inadecuados, insuficiente formación de los profesores, el no respeto de las orientaciones oficiales, la cuestión de lenguas en contacto y de la evaluación, entre muchos.

En cuanto a la evaluación del aprendizaje de español propiamente dicho, solo los estudios de Avome Mba (2007) y Obolo Omanda (2010) centran su problemática sobre la prueba escrita de español en el Bachillerato. La investigación de Avome Mba (2007:27) enfatiza en que la tipología de actividades desarrolladas en las aulas no obedece a las orientaciones oficiales y, como consecuencia, la falta de congruencia entre la programación, la puesta en práctica y la evaluación. En otras palabras, no existe una relación estrecha entre lo que los profesores programan, enseñan y evalúan. De donde la necesidad de una alternativa o redefinir las modalidades de evaluación o rehacer los objetivos pedagógicos. Y como propuestas que se han de llevar a cabo, Avome Mba (2007) enfatiza:

Para que los alumnos realicen con mucha eficacia la prueba escrita del español, es necesario introducir desde la clase de seconde, apartados como:

- La ortografía, para dar herramienta o reglas de escritura de las palabras;

- El vocabulario, para que no se limiten ellos a la mera explicación de palabras difíciles, sino que se les dé la posibilidad de aprender sinónimos, antónimos, parónimos, etc. Eso ayudará o facilitará la realización del ejercicio de sustitución en la prueba escrita de español del Bachillerato. (p. 29) Obolo Omanda (2010), por su parte, pinta un cuadro de la situación de los resultados de Bachillerato muy preocupante:

En 2007, les provinces de l'Estuaire, du Haut-Ogooué et du Woleu-Ntem ont enregistré 7483 candidats en espagnol (LV1) pour un taux de réussite de 18,96\%. En 2008, 8704 sont inscrits dans ces trois provinces pour un taux de 17,56\%. Enfin, l'année 2009 enregistre 9233 candidats pour un taux de 34,93\%. A côté de ces faibles résultats, la proportion d'échec, est d'autant plus élevée qu'elle devrait interpeler l'Inspecteur Pédagogique. En effet, il est si alarmant qu'il devrait réagir: en 2007 , il est de $81,04 \%$; en 2008 , il se situe à $82,44 \%$ et en 2009 , il connait une baisse et se stabilise à $65,70 \%$. (p. 2) 
Este autor enfocó su estudio hacia la mejora de la prueba escrita, es decir buscó responder a la pregunta ¿cómo mejorar la prueba escrita de español para que sea interesante y darle sentido a los ojos de los candidatos? Para este autor, el problema mayor está en la longitud de la prueba y en el proceso de preparación de los alumnos que considera inadecuado al espíritu del examen escrito final. La contribución de este estudio fue proponer una nueva configuración de la PEE coincidiendo con la reforma de la PEE en el Bachillerato de 2011 (nota $\mathrm{N}^{\circ}$ 002/2011).

Pese a estos estudios, no hemos encontrado investigaciones que tengan como objetivo determinar si el diseño e implementación de las pruebas de entrenamiento se ajustan a la normativa que regula la PEE. El presupuesto de partida de esta investigación es que existen diferencias en el entrenamiento de los alumnos en función del perfil y de las prácticas del centro, dejando así a algunos en situación de desventaja. Así, desde una perspectiva comparativa, este estudio resulta una vía aún no explorada que puede contribuir a enriquecer la reflexión y forma de mejorar tanto el desempeño de los alumnos en las pruebas de Bachillerato como la práctica de la lengua española en Gabón.

\section{METODOLOGÍA}

\subsection{MÉTODO UTILIZADO Y PERTINENCIA}

Como este estudio sobre el desempeño de los alumnos en la PEE en el Bachillerato se enmarca en la investigación educativa se entiende que éste persigue comprender, conocer y explicar la realidad educativa, como base para construir la ciencia y desarrollar el conocimiento científico de la educación (Bisquerra, 2009, p. 37). En este sentido, esta investigación de carácter comparado, no experimental se engloba en un marco metodológico cualitativo y descriptivo. Como señalan comparatistas eminentes, la utilización del método comparativo requiere, al igual que cualquier otro método de análisis empírico, una serie de decisiones previas referidas al diseño de investigación. Conviene, entonces, en esta primera parte de la metodología definir y explicar la metodología comparativa aplicada a la educación.

Según el diccionario de la Real Academia Española (2014), comparar consiste en "(...) fijar la atención en dos o más objetos para descubrir sus relaciones o estimar sus diferencias". En este sentido, comparar supone establecer una relación entre dos o más cosas. Comparar refiere también a la acción de examinar o analizar dos o más objetos para luego poder establecer las diferencias y las semejanzas que mantienen entre sí. Lo que implica tener una comprensión y un conocimiento de la realidad objeto de comparación. Caballero et al. (2016:40) definen la comparación como "(...) el estudio o la observación de dos o más objetos, fenómenos o acontecimientos para descubrir sus relaciones o estimar sus semejanzas y sus diferencias". En la vida diaria usamos constantemente la comparación para resolver problemas de todo tipo en diversos contextos. 
La elección de la metodología comparada para este estudio se considera necesaria para poder establecer la relación entre las diferentes pruebas de entrenamiento diseñadas y aplicadas en cuatro institutos, así como para relacionarlas con la normativa que regula la PEE. Desde este propósito, este estudio cumpliría con las propiedades básicas de la comparación reseñadas en García Garrido (1996:131133):

a) Carácter fenomenológico, puesto que el objeto de la investigación tiene una naturaleza que ha permitido que se le pudiera observar a través de documentos. Y como indica García Garrido (1996:131), "(...) en principio, toda comparación se ejerce sobre hechos o aspectos observados u observables."

b) Pluralidad, puesto que el acercamiento al objeto de estudio ha sido a través de cuatro unidades de análisis: 4 centros representativos del sistema educativo. Como advierte García Garrido (1996:132), "(...) para que exista comparación, hace falta que existan al menos dos hechos o fenómenos."

c) Homogeneidad, puesto que se ha estudiado el mismo nivel educativo (bachillerato), con los mismos instrumentos. En palabras de García Garrido (1996:132), "(...) para que exista comparabilidad, que es lo mismo que decir posibilidad de comparación, es preciso que haya homogeneidad entre los fenómenos que se comparan. Homogeneidad es tanto como decir semejanza en sus rasgos constitutivos, en sus principios esenciales."

d) Globalidad, puesto que todos tienen elementos en común, entre otros: la preparación de los alumnos y su participación en el examen de bachillerato. Desde esta perspectiva, García Garrido (1996:133) considera que "(...) la globalidad vendría exigida incluso, como vemos, si el objeto de comparación fuera un solo fenómeno (un solo sistema educativo, un solo aspecto del mismo, etc.".

La Educación Comparada (EC) es una ciencia, con objeto propio y metodología propia pero que necesita el aporte de un vasto conjunto de disciplinas (García Garrido, 1996, p. 138-139). Es también considerada como herramienta de apoyo para mejorar los sistemas educativos, comprenderlos y proponer reformas que permitan eliminar los problemas que los caracterizan. Como ciencia, la EC ha asistido a un considerable número de innovaciones metodológicas, generando discusiones y desacuerdos perceptibles en la existencia de "(...) varios enfoques metodológicos, cada uno de ellos con una variada secuela de recursos propios" (García Garrido, 1996, p. 113).

\subsection{DELIMITACIÓN DE LA INVESTIGACIÓN}

\section{Delimitación del objeto}

Cuando se usa el método comparativo de investigación conviene en sus inicios, además de definir el método concreto utilizado, delimitar el objeto del estudio. En palabras de García Garrido (1996:153), 
se debe delimitar la "(...) realidad o parcela de la realidad educativa que va a someterse a estudio". Tomando en cuenta la configuración del objeto de estudio, la PEE, la realidad es triple:

a) La referida a la estructura general de la PEE;

b) La referida a los bloques de contenidos;

c) La referida a la puntuación y ponderación

Así que, los cuatro centros que constituyen las unidades de análisis son:

1. Liceo Nacional Léon Mba (LNLM): instituto público, uno de los pioneros del sistema educativo.

2. Liceo Paul Indjendjet Gondjout (LPIG): uno de los numerosos institutos públicos de Gabón con una capacidad de ingreso enorme.

3. Liceo Paul Emane Eyegué (LPEE): otro de los institutos públicos con mayor alumnado.

4. Institución Inmaculada Concepción (IIC): instituto católico de renombre.

La justificación de la selección de estos institutos desde la metodología comparada se fundamentó en que todos:

- Tienen una larga tradición educativa y una experiencia en la preparación de los alumnos para las pruebas de Bachillerato;

- Poseen las trayectorias A1, A2 y B cuyos alumnos se examinan en E/LE;

- Constituyen el grupo de los centros que obtienen más admitidos en las pruebas de Bachillerato en todo el país;

- Tienen a profesores formados en la Escuela Normal Superior y participan activamente en los seminarios y talleres organizados por los organismos educativos.

Consideramos que los cuatro institutos (LNLM, LPIG, LPEE, IIC) forman una muestra bastante representativa del conjunto de los centros del país, puesto que todas las pruebas de Bachillerato son concebidas y elaboradas en Libreville, la capital. Además, algunos de los profesores de estos centros elaboran pruebas que son almacenadas en los bancos de datos de la "Direction du Baccalauréat". La mayoría de los centros del país utilizan las pruebas de entrenamientos de los centros de referencia para preparar a sus alumnos. Cabe añadir que la muestra seleccionada constituye el núcleo de los institutos con los que colaboran las autoridades para llevar a cabo sus actividades de información, formación y control. Pero señalamos una forma de marginalización de los demás centros, y sobre todo los que se sitúan en el interior del país cuyo acceso y estructuras hacen difícil el día a día de los docentes y del personal administrativo. 


\section{Delimitación del método}

En las fases de la investigación comparada, es una obligación desde el principio "(...) establecer de antemano los recursos metodológicos con los que podrá o deberá contarse" (García Garrido, 1996, p. 154). En lo que se refiere a la delimitación del método comparativo utilizado, se consideraron dos técnicas. Para atender al primer objeto de estudio, la estructura de la PEE, se revisó la normativa que establece los estudios de Bachillerato y que regula la PEE. Para este previo paso, se utilizaron las publicaciones oficiales sobre leyes que rigen la Educación y la Enseñanza en la República de Gabón por una parte; y por otra, la normativa que establece la Evaluación del Baccalauréat y que organiza la estructura del examen.

Por otro lado, para responder al segundo y tercer objetos de estudio, se utilizaron documentos recogidos en cada instituto. Estos documentos, esencialmente, se componen de pruebas de entrenamiento y otra categoría de evaluaciones diseñadas y aplicadas en cada centro. Como toda institución, los centros de enseñanza generan información periódicamente que van almacenando en sus bancos de datos. Y con el tiempo, esta información se convierte en valiosa para el trabajo metodológico ya que se constituye en datos interesantes sobre los cuales empezamos a depurar la información necesaria.

En el marco de esta investigación, el acceso a los documentos que sirven para preparar a los alumnos de bachillerato ha sido muy relevante. El desarrollo de las pruebas de entrenamiento constituye una fase importante del desempeño de los alumnos de bachillerato. Su organización y preparación parece mucho al examen final: los alumnos están siendo evaluados en todas las materias del currículo en función de las diferentes especialidades. También, los profesores de instituto suelen diseñar e implementar diferentes evaluaciones para valorar el nivel de adquisición de competencia lingüística de sus alumnos de bachillerato, con el objetivo de ir preparándoles para el examen escrito de Bachillerato.

Las evaluaciones que más se practican en el aula gabonés de E/LE son: evaluación diagnóstica, de aprovechamiento y sumativa. La evaluación formativa ocupa poco lugar por la simple razón que el currículo prescritivo obliga a los docentes a medir sistemáticament el grado de asimilación de los objetivos específicos por sus alumnos (la programación). Por eso, los docentes "(...) están más preocupados por medir la retroalimentación a los alumnos que valorar lo que saben hacer con la lengua" (Ambomo, 2019, p 153)

\subsection{ESTUDIO DESCRIPTIVO}

Como se ha anunciado, la fase analítica de este estudio se llevó a cabo mediante la realización de diferentes tareas. En primer lugar, se recopilaron los datos relacionados con la normativa que regula los estudios de bachillerato y que establece la estructura de la prueba escrita de español. Para su obtención, se acudió a las publicaciones del estado. A través de sus diversas instituciones, el estado gabonés realiza 
análisis de sus sectores de forma que permiten al investigador científico utilizar estos datos en beneficio de futuras investigaciones. Por ser el estado, las fuentes adquieren carácter oficial y de seriedad. García Garrido (1996:183) incluye en esta categoría de documentos "(...) todos aquéllos elaborados y publicados (aunque sea en ediciones restringidas) por las autoridades gubernativas y referentes, como es obvio, al propio sistema educativo o a alguno de sus aspectos".

Además, la colaboración con algunos profesores de instituto y un consejero pedagógico facilitó el acceso a varios textos oficiales que fundamentan la E/A de E/LE en el sistema educativo de Gabón. También durante el curso 2014-2015, se solicitó y se obtuvo de la "Direction du Baccalauréat" el acceso a las producciones escritas de los alumnos de español en la prueba de acceso a la universidad de la convocatoria de julio 2013. También, pudimos recolectar las estadísticas de los resultados obtenidos por los alumnos en la PEE de las trayectorias A1, A2 y B en el Bachillerato de cuatro convocatorias: de 2010 a 2013.

Luego, se revisó la normativa que establece la estructura de la prueba escrita de español. La PEE está concebida para evaluar destrezas básicas, tales como la comprensión de textos; la capacidad de análisis, relación y síntesis; la expresión de ideas; la valoración crítica y la demostración de conocimientos sobre contenidos relacionados con la lengua y la cultura general. La PEE se estructura en tres (3) bloques de contenidos: I. Comprensión del texto, II. Expresión personal, III. Competencia lingüística, y exige de los candidatos una fuerte movilización de su saber y saber hacer.

De forma paralela, se analizaron cuatro (4) pruebas de entrenamiento que corresponden al curso académico 2012-2013, en función de la estructura, bloques de contenidos, puntuación otorgada y ponderación. Anualmente, cada instituto organiza pruebas de entrenamiento en todas las materias del currículo para preparar a sus alumnos al examen de Bachillerato. Estas pruebas se suelen llamar "bachillerato de prueba". Los profesores de cada departamento diseñan pruebas que se aplican en su instituto y suelen intercambiarse entre centros constituyendo así una forma de compartir prácticas educativas. Una vez realizadas estas acciones previas, con los materiales recogidos se pasó al análisis formal, material y explicativo de éstos.

\subsection{ESTUDIO COMPARATIVO}

García Garrido (1996:159) señala que "(...) la tarea más propia de esta fase no es precisamente analítica, sino sintética; no es descomponedora de un todo en sus partes, sino componedora de las partes en un todo comparativo armónico". La fase sintética se compone de dos partes que coinciden: la primera, con la yuxtaposición y comparación, en la que se confrontan los datos y se comparan; y con las conclusiones comparativas, la segunda. Para la selección de datos y de conclusiones analíticas, se diseñó 
una pauta con unidades de comparación. Con el material seleccionado en el paso precedente, mediante tablas se llegó a yuxtaponer la información en relación a la estructura, bloques de contenidos, puntuación y ponderación. Este paso de yuxtaposición de datos permitió ver si las características de las pruebas que se comparan se corresponden totalmente, en parte o no se corresponden. Una vez realizado este paso, ya se pudieron emitir las conclusiones comparativas.

\section{RESULTADOS}

El análisis de las pruebas de entrenamiento diseñadas y aplicadas en 4 institutos, reveló que todos evalúan tres bloques de contenidos. Entre un número de materiales didácticos utilizados los textos literarios, periodísticos, publicitarios etc., ya forman parte de los materiales didácticos de uso frecuente en las aulas de lenguas. Los cuatro textos presentados son de diferente tipología: narrativo, descriptivo, expositivo, argumentativo. El número de líneas de cada texto tampoco se corresponde: varía entre 27 y 40 líneas. La duración de la prueba es de 3 horas para todas las pruebas.

Tabla 3.1. Yuxtaposición de datos de los textos presentados por los 4 centros educativos del estudio

\begin{tabular}{|c|c|c|c|c|}
\hline \multicolumn{5}{|c|}{ Características de las pruebas presentadas } \\
\hline CENTROS & LNLM & LPIG & LPEE & IIC \\
\hline $\begin{array}{l}\text { Tipología } \\
\text { textual }\end{array}$ & $\begin{array}{l}\text { Narrativo } \\
\text { (novela) }\end{array}$ & $\begin{array}{l}\text { Descriptivo } \\
\text { (reportaje) }\end{array}$ & $\begin{array}{c}\text { Expositivo } \\
\text { (artículo divulgativo) }\end{array}$ & $\begin{array}{l}\text { Argumentativo } \\
\text { (discurso) }\end{array}$ \\
\hline $\begin{array}{l}\text { Fuente y fecha } \\
\text { de publicación }\end{array}$ & $\begin{array}{l}\text { Un peso en } \\
\text { el mundo } \\
\text { (1999) }\end{array}$ & $\begin{array}{c}A B C \\
25 / 03 / 2013\end{array}$ & $\begin{array}{l}\text { ¡Despertad! } \\
\text { 12/2012, pp. 3-7 }\end{array}$ & $\begin{array}{c}\text { No hay fuente }_{4} \\
\text { 03/08/2009 }\end{array}$ \\
\hline Título & $\begin{array}{l}\text { Me tocaba } \\
\text { llegar lejos }\end{array}$ & $\begin{array}{l}\text { El Papa Francisco } \\
\text { convoca a los Jóvenes } \\
\text { en la JMJ de Río de } \\
\text { Janeiro }\end{array}$ & La impaciencia & $\begin{array}{l}\text { El } 30 \text { Aniversario del } \\
\text { Golpe de la Libertad en } \\
\text { Guinea Ecuatorial }\end{array}$ \\
\hline $\begin{array}{l}\text { Tipo de } \\
\text { material }\end{array}$ & Auténtico & $\begin{array}{l}\text { Manipulado: } \\
\text { desde el título hasta el } \\
\text { orden } \\
\text { de los párrafos } \\
\text { (supresión } \\
\text { de algunos) } \\
\end{array}$ & $\begin{array}{l}\text { Manipulado: } \\
\text { desde el título hasta el } \\
\text { orden } \\
\text { de los párrafos } \\
\text { (supresión } \\
\text { de algunos) } \\
\end{array}$ & $\begin{array}{l}\text { Manipulado: } \\
\text { desde el título } \\
\text { hasta el orden } \\
\text { de los párrafos } \\
\text { (supresión } \\
\text { de algunos) } \\
\end{array}$ \\
\hline $\begin{array}{l}\text { Número de } \\
\text { líneas }\end{array}$ & 27 & 40 & 35 & 36 \\
\hline
\end{tabular}

\footnotetext{
${ }_{4}$ La versión completa del discurso del Presidente Obiang Nguema de 03/08/2009 fue publicado en la página web institucional
} de Guinea Ecuatorial (https://www.guineaecuatorialpress.com/noticia.php?id=93)del) 
Palabras

explicadas
5

$3 \mathrm{~h}$
12

$3 \mathrm{~h}$
8

6

Duración

$3 h$

$3 \mathrm{~h}$

$3 h$

Al observar los datos de los textos presentados por los 4 institutos, se nota un fuerte predominio de textos periodísticos (3) en detrimento de los literarios (1). La yuxtaposición de esta categoría de elementos revela diferencias sustanciales entre las pruebas presentadas. Al nivel del número de líneas, se nota que el texto presentado por el LNLM (27 líneas) no está en adecuación con la normativa que sugiere entre 35 y 40 líneas. En cuanto al número de palabras explicadas, sólo un instituto, LPIG, coincide con la norma (12 palabras). Los tres otros, LNLM (5 palabras), IIC (6 palabras) y LPEE (8 palabras), no aplican la normativa. Como puede apreciarse en la tabla, los textos en su mayoría han sido manipulados por los equipos docentes de tres centros: LPIG, LPEE e IIC. La manipulación va de la sustitución de título a la supresión de varios párrafos para crear un nuevo texto.

\section{Datos del bloque 1. Comprensión del texto}

En la yuxtaposición de los datos del Bloque 1. Comprensión del texto (tabla 3.2), aparecen similitudes y diferencias sustanciales:

- Dos de los cuatro centros presentan 3 preguntas según establece la normativa (LNLM, IIC). En cuanto a los dos otros (LPEE y LPIG), presentan 4 preguntas.

- El análisis de los contenidos de las tareas a realizar revela la presencia de varias preguntas de "comentario crítico guiado" que exigen más esfuerzos de análisis por parte de los alumnos: LNLM (1), LPIG (2) y LPEE (1). El IIC es el único centro que presenta una tarea de "asignación del tema".

- El orden de presentación de las tareas también difiere de un centro a otro.

A pesar de una similitud aparente, se nota que las tareas a realizar en este bloque carecen de homogeneidad.

Tabla 3.2. Yuxtaposición y comparación de datos del bloque 1

\begin{tabular}{|c|c|c|c|c|c|}
\hline $\begin{array}{c}\text { Unidad de } \\
\text { comparación }\end{array}$ & $\begin{array}{c}\mathrm{N}^{\mathbf{o}} \\
\text { preguntas } \\
\end{array}$ & Tareas a realizar & $\begin{array}{c}\text { Tipo de } \\
\text { contenido }\end{array}$ & Puntuación & Ponderación \\
\hline NORMATIVA & 3 & $\begin{array}{l}\text { - Identificación de la } \\
\text { idea general } \\
\text { - Asignación del } \\
\text { tema } \\
\text { - Realización de un } \\
\text { resumen }\end{array}$ & $\begin{array}{l}\text { Saber } \\
\text { hacer }\end{array}$ & 5 & $\begin{array}{c}2 \\
1,5\end{array}$ \\
\hline
\end{tabular}




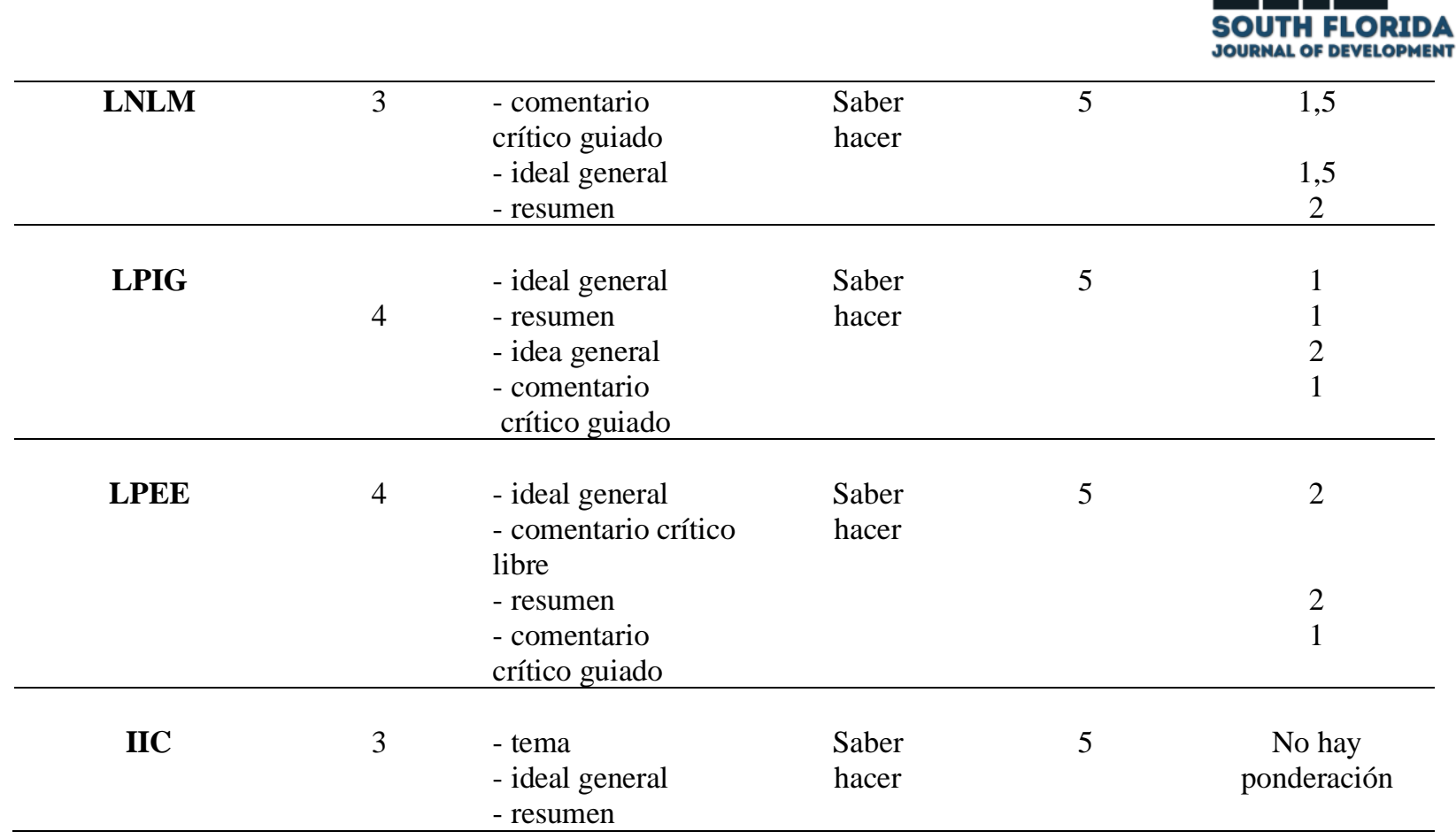

Al yuxtaponer las diferentes tareas a realizar del bloque 1 de las 4 pruebas, puede notarse que todas no coinciden con la normativa. En este bloque de contenidos, la frecuencia de las tareas a realizar deja aparecer algunos puntos de convergencia entre los institutos: las tareas "identificación de la idea general/principal" (5) y "realización de un resumen" (4) son las más requeridas. Pero, en las demás tareas, aparecen divergencias: "comentario crítico guiado" (3), "comentario crítico libre" (1) y "asignación del tema" (1). Dentro de esta categoría de tareas, las dos primeras no se requieren en comprensión del texto, sino en el bloque de expresión personal.

Figura 3.1. Yuxtaposición y comparación de tareas a realizar, bloque I

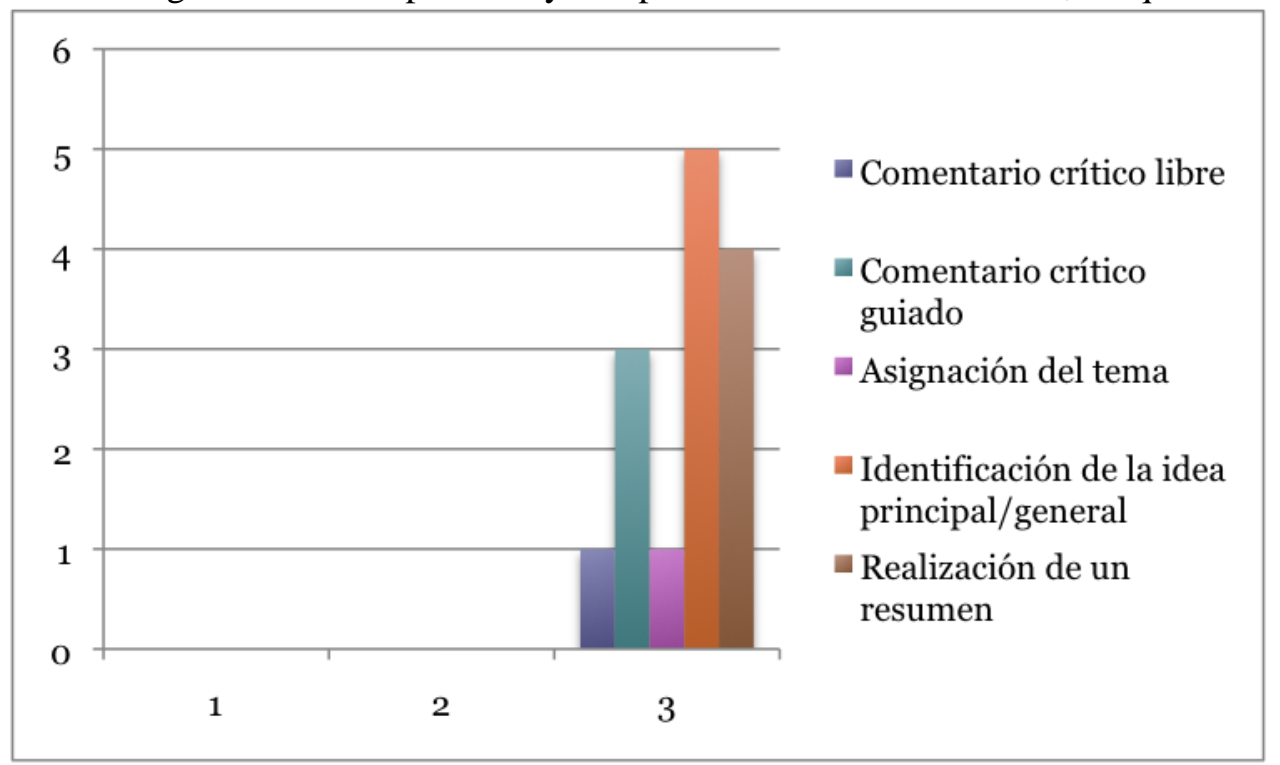




\section{* Datos del bloque 2. Expresión Personal}

Cuando se yuxtaponen y se comparan los contenidos de las tareas a realizar en el bloque 2 Expresión personal aparecen similutudes entre los centros. LPIG y LPEE presentan una pregunta de "comentario guiado personal" y otra de "comentario guiado libre", con una diferencia en el orden de presentación. LNLM presenta una de "valoración personal" y otra de "comentario crítico libre". En cuanto al IIC aparece una de "comentario crítico guiado" y otra de opinión personal.

Tabla 3.3. Yuxtaposición y comparación de datos del bloque 2

\begin{tabular}{|c|c|c|c|c|c|}
\hline $\begin{array}{c}\text { Unidad de } \\
\text { comparación }\end{array}$ & $\begin{array}{c}\mathrm{N}^{\mathbf{0}} \\
\text { preguntas }\end{array}$ & $\begin{array}{l}\text { Tareas a } \\
\text { realizar }\end{array}$ & $\begin{array}{c}\text { Tipo de } \\
\text { contenido }\end{array}$ & Puntuación & Ponderación \\
\hline NORMATIVA & 2 & $\begin{array}{l}\text { - comentario crítico } \\
\text { guiado } \\
\text { - comentario crítico libre }\end{array}$ & Saber hacer & 6 & $\begin{array}{l}3 \\
3\end{array}$ \\
\hline LNLM & 2 & $\begin{array}{l}\text { - comentario crítico } \\
\text { guiado } \\
\text { - comentario crítico libre }\end{array}$ & Saber hacer & 7 & $\begin{array}{c}\text { No hay } \\
\text { ponderación }\end{array}$ \\
\hline LPIG & 2 & $\begin{array}{l}\text { - comentario crítico } \\
\text { guiado } \\
\text { - comentario crítico libre }\end{array}$ & Saber hacer & 6 & $\begin{array}{l}3 \\
3\end{array}$ \\
\hline LPEE & 2 & $\begin{array}{l}\text { - comentario crítico } \\
\text { guiado } \\
\text { - comentario crítico libre }\end{array}$ & Saber hacer & 6 & $\begin{array}{l}3 \\
3\end{array}$ \\
\hline IIC & 2 & $\begin{array}{l}\text { - comentario crítico } \\
\text { guiado } \\
\text { - comentario crítico libre }\end{array}$ & Saber hacer & 6 & $\begin{array}{c}\text { No hay } \\
\text { ponderación }\end{array}$ \\
\hline
\end{tabular}

\section{Datos del bloque 3. Competencia lingüística}

La yuxtaposición de los datos del bloque 3 de los cuatros centros hace aparecer puntos de convergencia a la hora de atribuir los puntos. Tres de los centros (LPIG, LPEE, IIC) tienen la misma puntuación (9 puntos). No obstante, se evidencian divergencias notables a la hora de distribuir los puntos entre las diferentes tareas a realizar. Todas las distribuciones no coinciden con lo que establece la norma. Del mismo modo uno de los centros no ha previsto ninguna ponderación. Mientras que el LNLM presenta una distribución diferente ( 8 puntos)

Tabla 3.4. Yuxtaposición y comparación de datos del bloque 3

\begin{tabular}{|c|c|c|c|c|c|}
\hline $\begin{array}{l}\text { Unidad de } \\
\text { comparación }\end{array}$ & $\begin{array}{c}\mathrm{N}^{\mathbf{0}} \\
\text { preguntas }\end{array}$ & Tareas a realizar & $\begin{array}{c}\text { Tipo de } \\
\text { contenidos }\end{array}$ & Puntuación & Ponderación \\
\hline
\end{tabular}




\begin{tabular}{|c|c|c|c|c|c|}
\hline NORMATIVA & 5 & $\begin{array}{l}\text { - Transformar } \\
\text { - Imitar } \\
\text { - Sustituir } \\
\text { - Completar } \\
\text { - Traducir }\end{array}$ & $\begin{array}{c}\text { Saber } \\
y \\
\text { saber hacer }\end{array}$ & 9 & $\begin{array}{lr}2 & (1-1) \\
1 & (0,5-0,5) \\
1 & (0,5-0,5) \\
2 & (1-1) \\
3 & (2-1) \\
\end{array}$ \\
\hline LNLM & 5 & $\begin{array}{l}\text { - Transformar } \\
\text { - Imitar } \\
\text { - Sustituir } \\
\text { - Completar } \\
\text { - Traducir }\end{array}$ & $\begin{array}{c}\text { Saber } \\
\text { y } \\
\text { saber hacer }\end{array}$ & 8 & $\begin{array}{lr}1,5 & (1-0,5) \\
1,5 & \text { (No hay) } \\
1 & \text { (No hay) } \\
1,5 & \text { (No hay) } \\
2,5 & \text { (No hay) }\end{array}$ \\
\hline LPIG & 5 & $\begin{array}{l}\text { - Transformar } \\
\text { - Imitar } \\
\text { - Sustituir } \\
\text { - Completar } \\
\text { - Traducir }\end{array}$ & $\begin{array}{c}\text { Saber } \\
\text { y } \\
\text { saber hacer }\end{array}$ & 9 & $\begin{array}{lr}2 & (1-1) \\
1 & (0,5-0,5) \\
1 & (0,5-0,5) \\
2 & (1-1) \\
3 & (1,5-1,5) \\
\end{array}$ \\
\hline LPEE & 5 & $\begin{array}{l}\text { - Transformar } \\
\text { - Imitar } \\
\text { - Sustituir } \\
\text { - Completar } \\
\text { - Traducir }\end{array}$ & $\begin{array}{c}\text { Saber } \\
\text { y } \\
\text { saber hacer }\end{array}$ & 9 & $\begin{array}{cc}3 & (0,5-0,5) \\
1 & (0,5-0,5) \\
1 & (0,5-0,5) \\
1 & (0,5-0,5) \\
3 & (2-1)\end{array}$ \\
\hline IIC & 5 & $\begin{array}{l}\text { - Transformar } \\
\text { - Imitar } \\
\text { - Sustituir } \\
\text { - Completar } \\
\text { - Traducir }\end{array}$ & $\begin{array}{c}\text { Saber } \\
\text { y } \\
\text { saber hacer }\end{array}$ & 9 & $\begin{array}{c}\text { No hay } \\
\text { ponderación }\end{array}$ \\
\hline
\end{tabular}

Al yuxtaponer las consignas en este mismo bloque (tabla 3.5), se nota también la no conformidad en la formulación de las consignas de las tareas a realizar que varía de un instituto a otro.

Tabla 3.5. Yuxtaposición y comparación de las consignas del bloque 3

\begin{tabular}{ll}
\hline & \multicolumn{1}{c}{ Consignas tareas a realizar } \\
\hline & - Imitar: "Reutiliza las estructura subrayadas en otra frase con otro verbo que \\
& - según la lógica del texto" \\
& - Sustituir: "Reemplaza las estructuras subrayadas por otras equivalentes" \\
& - Completar: "Completa las frases siguientes según la lógica del texto" \\
& - Traducir: "Pasa al francés", "Pasa al español" \\
& - Imitar: "emplea la estructura subrayada en frase personal respetando la lógica \\
& del texto" \\
& - Traducir: "traduce en español" \\
\hline LNLM & - Imitar: "Reutiliza las expresiones subrayadas en frases personales respetando \\
& la lógica del texto" \\
\hline LIPIG & - Imitar: "Reutiliza las palabras subrayadas en una frase respetando el contexto" \\
& - Sustituir: "Remplaza las estructuras subrayadas por otras de mismo sentido" \\
& - Completar: "Completa las frases siguientes sin alterar el sentido" \\
\hline LPEE & - Imitar: "reutiliza las estructuras subrayadas en una frase personal según la \\
& lógica del texto" \\
& - Sustituir: "Sustituir por un equivalente las estructuras subrayadas" \\
& - Completar: "completar las siguientes frases según la lógica del texto"
\end{tabular}




\section{* Datos de puntuación y ponderación.}

Al yuxtaponer la puntuación del bloque 1, se notan similitudes en el número de puntos asignados: cinco puntos sobre veinte (5/20). La ponderación indica diferencias: tres centros (LNLM, LPIG y LPEE) distribuyen los puntos de forma diferente. excepto el IIC que no presenta ninguna ponderación. Ninguna de las distribuciones corresponde a la normativa (tabla 3.56). Como puede verse, en la ponderación arrojan las diferencias.

Por su parte, en el bloque 2 se aprecian similitudes entre tres centros (LPIG, LPEE y IIC) cuya puntuación obedece a la normativa (6/20). Mientras que el LNLM otorga una puntuación diferente (7/20). En cuanto a la ponderación, dos centros (LPIG y LPEE) presentan una misma distribución (3-3). Los dos otros (LNLM y IIC) no presentan ninguna ponderación.

En cuanto al bloque 3, se observan también convergencias entre tres centros (LPIG, LPEE y IIC) cuya puntuación otorgada es 9/20. Pero en uno de los institutos (LNLM), pueden apreciarse puntos de divergencia (8/20). La ponderación otorgada a este bloque, una vez más, es diferente en función del centro que elaboró la prueba:

- LNLM: 1,5-1,5-1-1,5-2,5.

- LPIG: 2-1-1-2-3.

- LPEE: 3-1-1-1-3.

- IIC: no presenta ninguna ponderación.

Estas divergencias observadas en la preparación de las pruebas muestran que los diferentes equipos docente no habían establecidos y diseñados todos los criterios de evaluación. Las divergencias notadas en la ponderación (tabla 3.6) hacen pensar en que probablemente los docentes no hayan recibido una formación igual o simplemente no han recibido ninguna formación en las técnicas de evaluación. Por lo que pueden apreciarse las carencias del profesorado en cuestiones de evaluación.

Tabla 3.6. Yuxtaposición y comparación de datos puntuación y ponderación

\begin{tabular}{ccccc}
\hline $\begin{array}{l}\text { Bloques de } \\
\text { contenidos }\end{array}$ & LNLM & LIPIG & LPEE & IIC \\
\hline \multirow{3}{*}{ Bloque I } & $\mathbf{5}$ & $\mathbf{5}$ & $\mathbf{5}$ & $\mathbf{5}$ \\
& 1,5 & 1 & 2 & No hay \\
& 1,5 & 1 & 2 & ponderación \\
\hline \multirow{3}{*}{ Bloque II } & 2 & 1 & 1 & $\mathbf{6}$ \\
& $\mathbf{7}$ & $\mathbf{6}$ & $\mathbf{6}$ & No hay \\
& No hay & 3 & 3 & ponderación \\
\hline Bloque III & $\mathbf{8}$ & 3 & 3 & $\mathbf{9}$ \\
\hline
\end{tabular}




\begin{tabular}{ccccc} 
& & & $\begin{array}{c}\text { SOUTH } \\
\text { JOURMAL of }\end{array}$ \\
\hline 1,5 & 1 & 1 & ponderación \\
1.5 & 1 & 1 & \\
2.5 & 2 & 1 & \\
\hline
\end{tabular}

También, pueden apreciarse puntos de convergencia entre tres institutos en relación a la ponctuación otorgada a cada bloque de contenidos. No obstante, existen numeros puntos de divergencia en cuanto a la ponderación en varias tareas.

Figura 3.1. Yuxtaposición y comparación de datos de la puntuación otorgada a cada bloque de contenidos

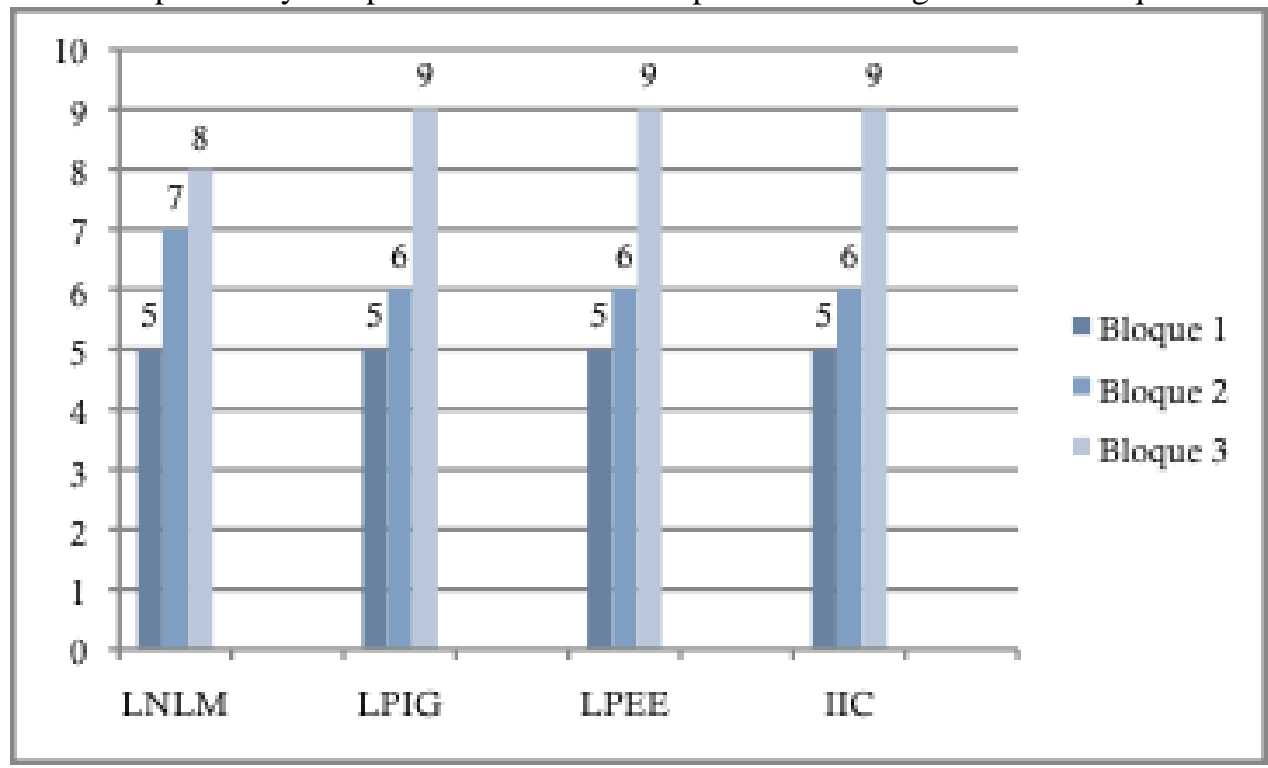

\section{DISCUSIÓN}

Tras analizar y caracterizar las cuatro pruebas de entrenamiento, la yuxtaposición de las conclusiones analíticas ha resaltado algunas similitudes en el número de preguntas presentadas (bloque 1. Comprensión del texto). Dos centros presentan 3 preguntas (LNLM, IIC) y los otros dos presentan cuatro y cinco preguntas (LPIG, LPEE). El análisis de los contenidos de las tareas a realizar revela la presencia de varias preguntas de "comentario crítico guiado" en las pruebas de tres centros (LNLM, LPIG y LPEE) y algunas divergencias a la hora de aplicar la normativa. Este tipo de tareas son recomendadas para el bloque 2 Expresión personal porque exigen un esfuerzo de análisis. El análisis de los contenidos de las tareas a realizar en este bloque mostró algunas divergencias.

La presencia de preguntas de análisis crítico en la comprensión del texto parece responder a una interpretación subjetiva de la norma, lo que incide sistemáticamente en la preparación de los alumnos para el desarrollo y la adquisición de esta destreza. Al analizar la puntuación en este bloque, se observó similitudes en el número de puntos asignados: cinco puntos sobre veinte (5/20). En la ponderación es dónde arrojan las diferencias. Cada centro distribuye los puntos de forma diferente, a excepción del IIC 
que no presenta ninguna ponderación. Ninguna de las distribuciones corresponde a la establecida en la nota $N^{o} 002$ (MEN, 2011).

Al comparar los datos del bloque 2 (tabla 3.3) puede verse que todos los centros presentan dos preguntas para valorar este bloque. Cuando se comparan los contenidos de las tareas a realizar aparecen algunas divergencias entre los centros. LPIG y LPEE presentan una pregunta de comentario crítico y otra de comentario crítico libre o personal, con una diferencia en el orden de presentación. LNLM presenta una de valoración personal y otra de comentario crítico personal. En cuanto al IIC aparece una de comentario crítico libre y otra de opinión personal. A pesar de una similitud aparente, se nota que las tareas a realizar en este bloque carecen de homogeneidad. Lo que lleva a pensar que las prácticas docentes de cada centro son manifiestas en el diseño de las pruebas de evaluación a pesar de la normativa.

La yuxtaposición de los datos del bloque 3 (tabla 3.4), pone de relieve que todos los centros presentan cinco preguntas y según el orden establecido por la normativa. Al comparar los contenidos de las tareas a realizar aparecen puntos divergentes en la formulación de las consignas en tres tareas. En imitar, ninguno de los cuatro centros coincide con la formulación de la norma. En completar 3 centros coinciden (LNLM, LPIG, IIC), excepto el LPEE. En sustituir las consignas tampoco coinciden todos los centros con la normativa. La presencia de estas diferencias en la formulación de las consignas puede hacer sospechar que en la práctica docente, los alumnos no están siendo entrenados adecuadamente en la adquisición de competencias procedimentales, "saber hacer".

También, al comparar la puntuación asignada al bloque 3 en la pruebas reaparecen diferencias que oscilan entre 8 y 9. El hecho de que en la preparación de las pruebas no se hayan establecido y diseñado todos los criterios para la calificación lleva a pensar que la prueba no garantiza la validez y la fiabilidad. Se ha notado que el nivel de dificultad varía de una prueba a otra y en función del tipo de texto presentado.

Los resultados obtenidos ponen de manifiesto la existencia de algunos puntos divergentes entre los institutos al aplicar la norma que establece la PEE en el Bachillerato. Las diferencias observadas en los contenidos de las tareas a realizar, así como en la puntuación y en la ponderación dejan pensar que la norma básica está sujeta a interpretaciones diversas por cada instituto en que se la aplique y parecen evidenciar las dificultades de los docentes en aplicar las recomendaciones oficiales. Como consecuencia de estas diferencias sustanciales, la falta de homogeneidad de las pruebas diseñadas y aplicadas en cada centro para entrenar a sus alumnos de bachillerato.

\section{CONCLUSIONES}

Esta investigación sobre el desempeño de los alumnos en la PEE en el Bachillerato ha tenido por objetivos analizar la prueba escrita de español y determinar si el diseño de ésta se ajusta a la normativa 
que la regula. De manera específica, hemos analizado y caracterizado las pruebas de entrenamiento diseñadas y aplicadas en 4 institutos con respecto a la normativa que establece los estudios de Bachillerato y que regula la estructura de la PEE (Estudio descriptivo). Una vez establecido los criterios de comparación y con los análisis realizados, se ha pasado a yuxtaponer y comparar las pruebas entre sí (Estudio comparativo).

El análisis de las 4 pruebas ha permitido extraer comparaciones acerca de la estructura, bloques de contenidos, puntuación y ponderación. La yuxtaposición de los resultados obtenidos del análisis de cada unidad de estudio evidencia diferencias sustanciales en la PEE en función del centro en el que se aplique la normativa.

De modo que en función del perfil y de las prácticas de cada instituto, el entrenamiento de los alumnos varía de un centro a otro. Por lo que los resultados alcanzados en la prueba pueden variar en un amplio margen puesto que todos los alumnos no tienen la misma situación de partida, lo que deja a muchos de ellos en situación de desventaja. Al ser común y obligatoria en todo el país, la PEE debería garantizar las mismas posibilidades de éxito a todos los candidatos. Lo que lleva a cuestionar tanto el proceso de preparación de los alumnos como la validez de las pruebas de evaluación diseñadas e implementadas por los docentes para conocer el nivel de dominio de adquisición de la lengua. Se considera que una prueba es válida cuando evalúa lo que se supone que evalúa (Sanmartí Puig, 2020). Tenemos razones para pensar que en el diseño de la PEE no se tiene en cuenta el nivel real de adquisición y desarrollo de las habilidades que se intentan evaluar, por consiguiente, no se están evaluando las habilidades que se pretenden evaluar (Bordón, 2015). 


\section{REFERENCIAS}

Ambomo, M-N. (2019). La evaluación del aprendizaje de lenguas extranjeras en los alumnos de la enseñanza secundaria: el caso de la enseñanza del español en Camerún (Tesis doctoral). Universidad de Lleida.

https://www.tesisenred.net/bitstream/handle/10803/668899/Tmna1de1.pdf?sequence=5\&isAllowed=y

Avome Mba, G. (2007). La prueba escrita del español en el bachillerato: hacia un estudio de modelos de enseñanza/aprendizaje del español en Gabón. En G. Nistal Rosique y G. Pié Jahn (Dirs.), La situación actual del español en África, Actas del II Congreso Internacional de Hispanistas en África (pp. 21-29). SIAL.

Bizqueara Alzina, R. (Ed.). (2009). Metodología de la investigación educativa. La Muralla.

Bordón Martínez, T. (2006). La evaluación de la lengua en el marco de E/LE: Bases y procedimientos. Arco Libros.

Bordón Martínez, T. (2015). La evaluación de segundas lenguas (L2). Balance y perspectivas. Revista Internacional de Lenguas Extranjeras, (4), 2015, 9-30.

Caro Muñoz, M. (2017). Pensamiento subyacente y enseñanza del español como lengua extranjera en el sistema de enseñanza reglada de Gabón (Tesis doctoral). Universitat de Barcelona. http://diposit.ub.edu/dspace/bitstream/2445/121859/1/MCM_TESIS.pdf

García Garrido, J. L. (1996). Fundamentos de Educación Comparada. (3ª Ed.). Dykinson.

Martín Perís, E. (2008). La autoevaluación: nuevas consideraciones sobre un viejo tema. En: S, Pastor Cesteros y S. Roca Marín (Ed.), La evaluación en el aprendizaje y la enseñanza del español como LE/ L2 (p. 27-44). Universidad de Alicante. https://repositori.upf.edu/bitstream/handle/10230/24633/mperis_asele2007_eval.pdf;sequence=1

Mbadinga Mbadinga, A-M. (2014). Représentations et stratégies d'enseignement / apprentissage de l'espagnol en milieu exolingue: Le cas des débutants gabonais (Tesis). Université Paris Ouest Nanterre La Défense.

Messakimove, S. (2021). Análisis de interferencias léxicas y morfosintácticas de las producciones escritas de alumnos de bachillerato de E/LE. South Florida Journal of Development (v.2, n.2), pp. 3461-3474.

Ministère de l'Éducation Nationale et de l'Instruction Civique. (2009). Séminaire de formation sur l'élaboration de l'épreuve écrite d'espagnol au baccalauréat.

Ministère de l'Éducation Nationale. (2011). Note $N^{o}$ 002/MEN/IG/ISDG/DE du 19 de décembre de 2011 portant modification de la note $N^{\circ} 53 / 05 / M E N / I G / I S D G / D E$ portant recommandation pour l'élaboration des sujets et la correction de l'épreuve écrite d'espagnol au baccalauréat.

Obolo Omanda, J. (2010). L'épreuve écrite d'espagnol au baccalauréat: Pour une redéfinition de l'épreuve (Memoria). IUFM de l'Académie de Lyon.

Pastor Cesteros, S. (2003). La evaluación del proceso de aprendizaje de segundas lenguas. En M.-V. Reyzábal Rodríguez (Dir.), Perspectivas teóricas y metodológicas: lengua de acogida, educación 
internacional y contextos inclusivos, 503-514. Comunidad de Madrid, Consejería de Educación. http://segundaslenguaseinmigracion.com/ense_anzal2/EvaluacionPastor.pdf

Real Academia Española (2014). Diccionario de la lengua española.

Sanmartí Puig, N. (2020). Evaluar y aprender: un único proceso. Octaedro Editorial. 